\title{
Metabolic and hormonal responses of low birthweight infants to intravenously infused calories not exceeding the maintenance energy expenditure
}

\author{
I. RUBECZ, J. MESTYÁN, P. VARGA, AND GY. SOLTÉSZ \\ Department of Paediatrics, University Medical School, Pécs, Hungary
}

SUMMARY Eight low birthweight infants were studied for their metabolic and hormonal responses to intravenous alimentation when the caloric input was varied. The study was made during three consecutive 12-hour periods, when solutions of glucose, glucose and amino-acids, and glucose and amino-acid and lipids were successively infused. Hypertonic glucose led to a fall in plasma free fatty acids and amino-acids. Supplementation with an amino-acid mixture increased the concentration of amino-acids but not above the fasting level. Thus, hypertonic glucose was an effective means for avoiding hyperaminoacidaemia. Blood urea was little changed, also indicating satisfactory use of amino-acids. The number and distribution of calories infused throughout the third 12-hour period, when lipid was infused, were also important factors contributing to the avoidance of hyperaminoacidaemia. The caloric needs of low birthweight infants kept at neutral temperatures should be individually assessed in the light of recent data. The administration of an unnecessarily large load of calories and nutrients to parenterally-fed infants can thereby be avoided.

Knowledge of the caloric expenditure of the newborn infant, and of the factors which may alter it, is essential when alimentation is given by the parenteral route. Thus, if preterm infants $<1500 \mathrm{~g}$ are fed intravenously using conventional estimates of maintenance energy expenditure, an excessive caloric load may be imposed leading to dangerous complications. These estimates often fail to take account of the fact that maintenance caloric requirement in the neonatal and postneonatal periods varies greatly according to weight, and gestational and postnatal ages (Mestyán et al., 1968; Rubecz et al., 1974; Rubecz and Mestyán, 1975). In view of this, we investigated the changes in the levels of the main plasma metabolites after infusion of nutritive mixtures with a low caloric value appropriate to the relatively low energy needs of low birthweight infants. The results may serve as a basis for further studies to define the caloric aspects of total parenteral nutrition in such infants.

Department of Paediatrics, University Medical School Pécs,

Hungary

I. RUBECZ, consultant physician

J. MESTYÁN, professor of paediatrics

P. VARGA, physician

GY. SOLTÉSZ, consultant physician

\section{Materials and methods}

Infants. Eight low birthweight infants (2 appropriate for and 6 small for gestational age), in whom early oral feeding had failed, were infused with nutritive solutions for 36 hours. The mean gestational age was 32 weeks (range 28-35) and the mean birthweight $1362 \mathrm{~g}$ (range 900-1930). The average postnatal age at which parenteral feeding began was 18 hours. Clinical data, the volume of the nutritive solutions, and the calories and amounts of glucose, amino-acids, and lipid infused are summarised in Table 1.

Infusions. The design of the short-term IV feeding was as follows: (1) $10 \%$ glucose was given for 12 hours; (2) during the second 12-hour period $10 \%$ glucose with $2.7 \%$ crystalline amino-acids (Aminosol 2400: Vitrum, Stockholm) was infused; (3) thereafter in addition to the glucose and amino-acid mixture, $10 \%$ lipid solution (Intralipid: Vitrum, Stockholm) was infused by a second pump connected through a Y-connection. In this third 12-hour period when a mixture of the three main nutrients was given, care was taken not to exceed appreciably the maintenance energy expenditure. The supplementation of glucose 


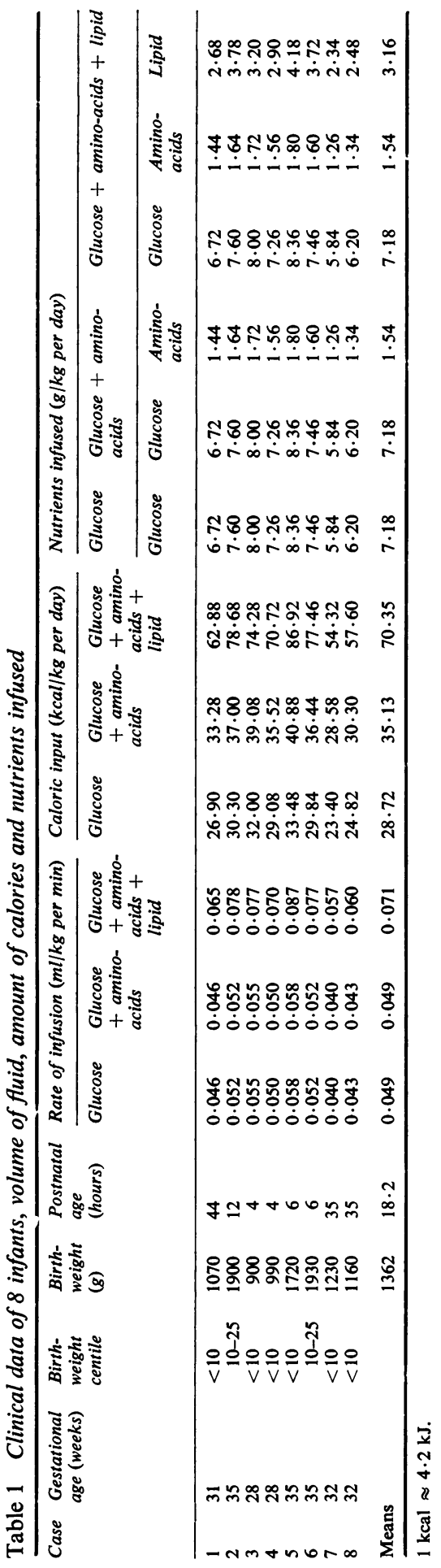

as the basic caloric source, first with amino-acids and then with fat emulsion, represented a short-term alimentation programme with increasing caloric input and changing composition of the nutritive mixture. In brief, the study consisted of three consecutive 12-hour periods during which solutions containing glucose, glucose and amino-acids, and glucose and amino-acids and lipids, were infused.

Chemical and hormonal monitoring. Blood samples were drawn through an umbilical venous catheter before IV nutrition and at the end of each infusion period. The following metabolites were measured: glucose (Pryce, 1967), free fatty acids (Dalton and Kowalski, 1967; Laurell and Tibbling, 1967), lactate (Huckabee, 1958), $\alpha$-amino-nitrogen (Rubinstein and Pryce, 1959), and urea (Ceriotti, 1971). In 6 infants the plasma concentrations of 17 individual amino-acids were also determined using a Beckman automatic amino-acid analyser. Plasma insulin and growth hormone were estimated (Hunter and Greenwood, 1962; Hales and Randle, 1963). Means and standard errors were calculated, and significance was estimated by the paired Student's $t$ test.

\section{Results}

Response of blood glucose, lactate, and plasma FFA. Fig. 1 shows that the concentrations of glucose at the end of the three infusion periods were higher than the control level $(P<0.01$ and $<0.05)$. The greatest rise was during the first 12-hour period when only $10 \%$ glucose was infused. Thereafter the addition of an amino-acid mixture to $10 \%$ glucose resulted in a fall in blood glucose, which remained still significantly above that of the preinfusion level $(\mathrm{P}<0 \cdot 05)$. Further supplementation with $10 \%$ lipid solution did not greatly influence blood glucose concentration which remained near the level of the end of the glucose and amino-acid period.

The mean concentration of plasma free fatty acids (FFA) fell in response to $10 \%$ glucose but, owing to the large individual variation of the fasting level, the fall was not significant. The infusion of amino-acids in addition to glucose caused a further decrease in the plasma FFA. Supplementation with $10 \%$ lipid solution, as expected, was associated with a pronounced rise in FFA concentration $(\mathrm{P}<0.01)$.

Mean blood lactate was slightly raised by the end of the $10 \%$ glucose period. Supplementation with amino-acids and fat emulsion induced a gradual fall in blood lactate. However, its concentration by the end of the third infusion period, owing to the large variation in the starting values, was again not significantly lower than that before IV nutrition. 

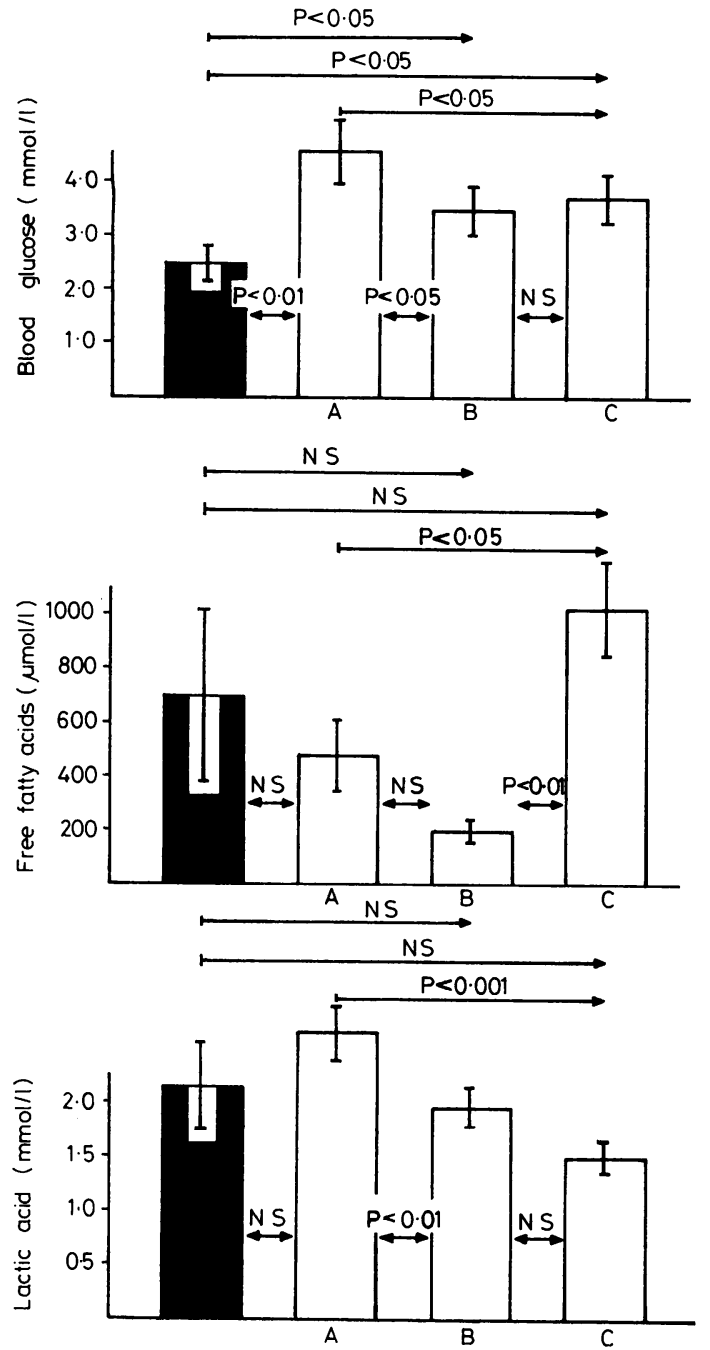

Fig. 1 Response of blood glucose, free fatty acids, and lactic acid to infusions of $(A) 10 \%$ glucose, (B) $10 \%$ glucose $+2.7 \%$ amino-acid, and $(C) 10 \%$ glucose $+2.7 \%$ amino-acid $+10 \%$ lipid.

Changes in the plasma $\alpha$-amino-nitrogen, urea, and amino-acid pattern. The changes in $\alpha$-amino-nitrogen and urea content of the plasma are shown in Fig. 2. The mean level of $\alpha$-amino-nitrogen fell significantly in response to $10 \%$ glucose $(P<0 \cdot 05)$, but regained the fasting level when an amino-acid mixture was added to glucose. Further supplementation with $10 \%$ lipid solution did not affect the level, which remained at the fasting value.

Mean urea nitrogen was not greatly affected, with only a slight tendency to rise during the second and
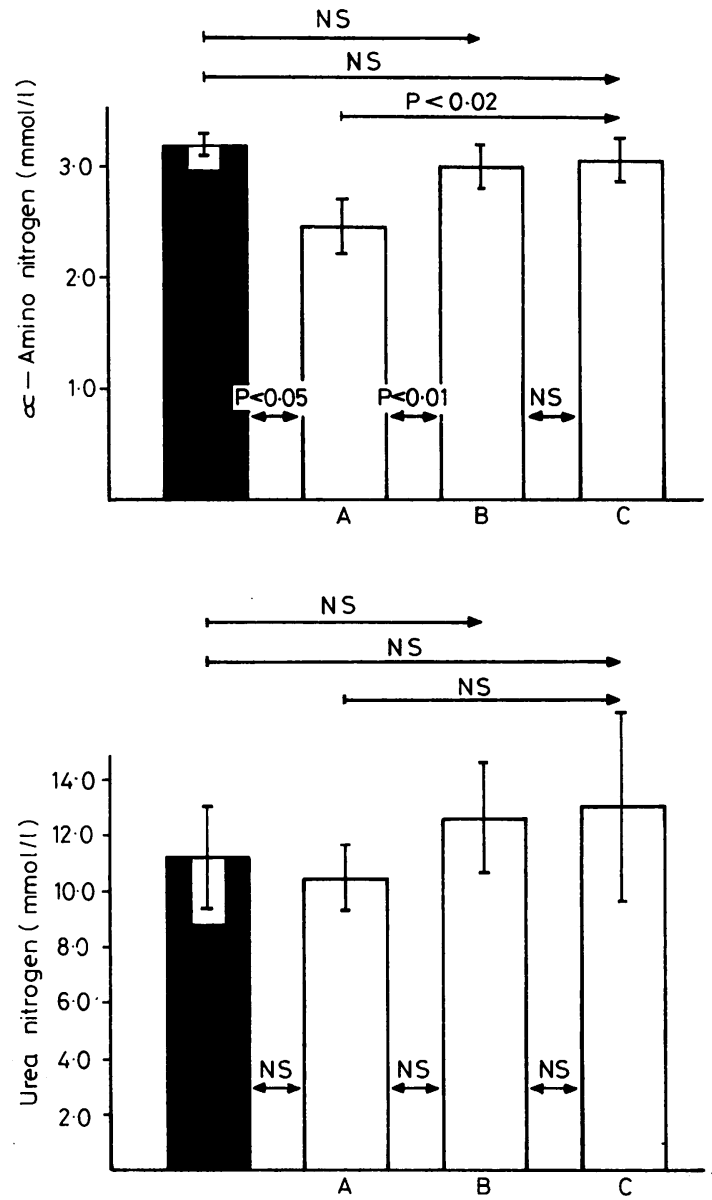

Fig. 2 Plasma $\alpha$-amino-nitrogen and urea nitrogen during infusion of $(A) 10 \%$ glucose, (B) $10 \%$ glucose $+2.7 \%$ amino-acid, and $(C) 10 \%$ glucose + $2.7 \%$ amino-acid $+10 \%$ lipid.

third 12-hour periods when the amino-acid mixture was given.

Unfortunately only 2 of the 6 infants in whom there were changes in the plasma concentration of 17 different amino-acids were tested before parenteral nutrition. However, from previous observations (Rubecz et al., 1974) we know that glucose infusion causes a pronounced fall in most individual aminoacids, and the same response of the plasma $\alpha$-aminonitrogen was seen in our study. The hypoaminoacidaemic effect of $10 \%$ glucose is also evident if the combined fasting concentration of the 17 aminoacids obtained in the 2 infants $(2673 \mu \mathrm{mol} / \mathrm{l})$ is compared with that obtained by the end of the $10 \%$ glucose period in 6 infants $(1559 \mu \mathrm{mol} / \mathrm{l})$. The 


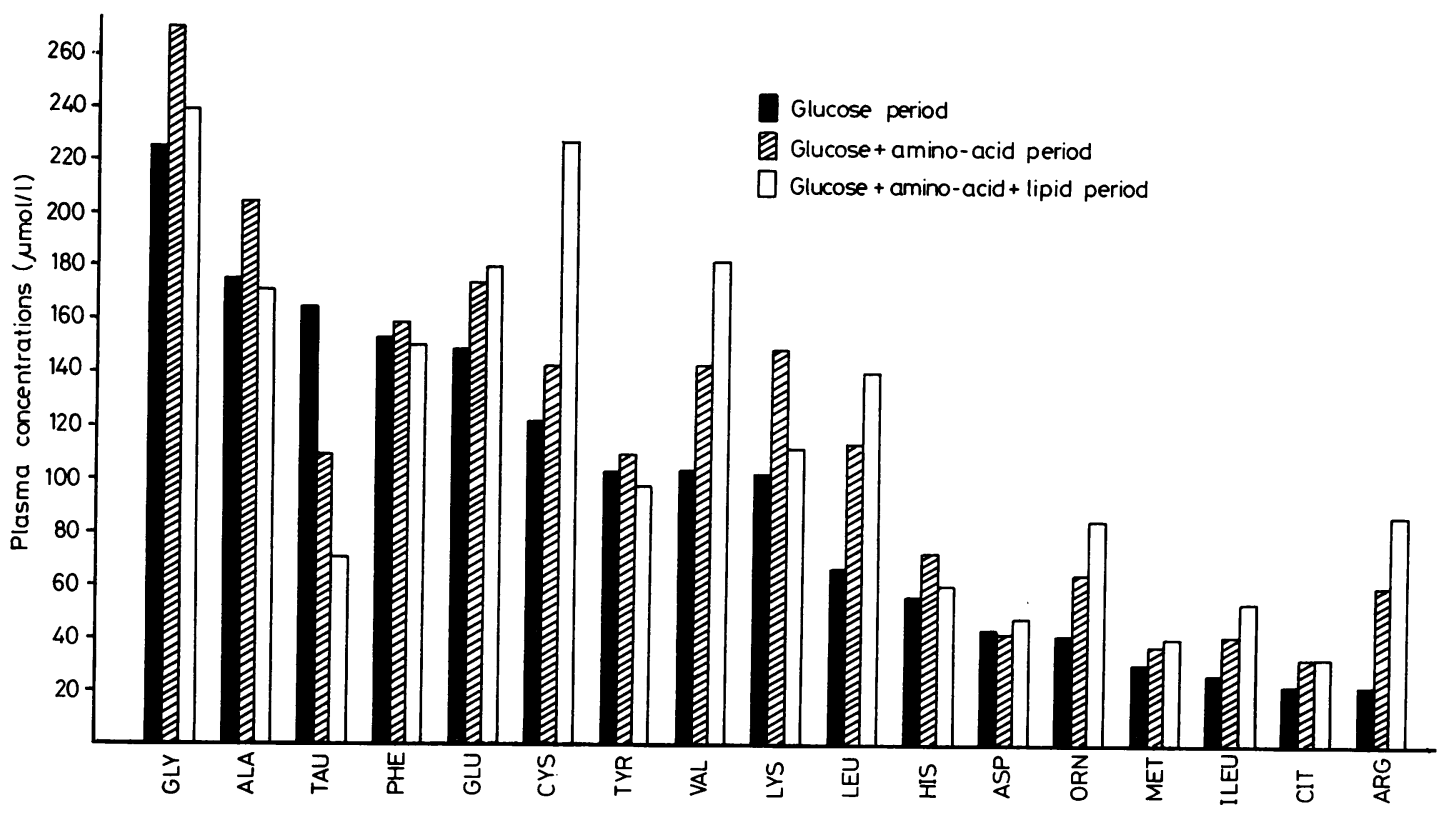

Fig. 3 Concentrations of individual plasma amino-acids during the three intravenous nutrition regimens.

responses of the individual amino-acids in the second and third 12-hour periods of intravenous nutrition are shown in Fig. 3. Owing to the small number of infants in whom each of the 17 amino-acids could be followed throughout the three infusion periods, statistical analysis was not made, but 13 amino-acids clearly increased their plasma concentrations when glucose was supplemented with amino-acid solution. The rise of phenylalanine, tyrosine, and asparagine was negligible. It is interesting that one amino-acid, taurine, responded to the addition of the amino-acid by a pronounced fall.

By the end of the third infusion period, when fat was added to the glucose and amino-acid mixture, a further rise was observed in the levels of 8 aminoacids (glutamine, cystine, valine, leucine, ornithine, methionine, isoleucine, and arginine), with no change or fall in the concentration of glycine, alanine, tyrosine, lysine, histidine, asparagine, citrulline, and phenylalanine. Among those amino-acids whose concentration decreased in response to lipid, in none did the concentration fall below that at the end of the first infusion period. Taurine, however, showed a further pronounced fall when glucose and amino-acid was supplemented with lipid.

Insulin and growth hormone response. The mean responses of plasma insulin and growth hormone (GH) are shown in Table 2. By the end of the $10 \%$ glucose period the mean plasma level had increased. The addition of amino-acid mixture to the infusate caused a further increase in the mean level of both hormones. By the end of the third period plasma insulin and $\mathrm{GH}$ mean levels were both lower.

It should be noted that the magnitude and the time course of individual insulin response (Fig. 4) showed

Table 2 Effect of glucose, glucose + amino-acid, and glucose + amino-acid + lipid infusion on plasma growth hormone and insulin concentration

\begin{tabular}{|c|c|c|c|c|c|}
\hline & & $\begin{array}{l}\text { Before intravenous } \\
\text { infusion }\end{array}$ & After $10 \%$ glucose & $\begin{array}{l}\text { After } 10 \% \text { glucose } \\
+2.7 \% \text { amino-acid }\end{array}$ & $\begin{array}{l}\text { After } 10 \% \text { glucose } \\
+2.7 \% \text { amino-acid } \\
+10 \% \text { lipid }\end{array}$ \\
\hline Insulin $(\mu U / m l)$ & Mean $\pm \mathbf{S E}$ & ${ }^{9.7} \pm 2.2 \quad P<0.05$ & $20 \cdot 3 \pm 5 \cdot 4$ & $29.8 \pm 13.6$ & $24 \cdot 7 \pm 11 \cdot 9$ \\
\hline Growth hormone (ng/ml) & Mean $\pm \mathbf{S E}$ & $\begin{array}{r}19.0 \pm 2.1 \\
P<0.01\end{array}$ & $51 \cdot 6 \pm 7 \cdot 1$ & $65 \cdot 2 \pm 4 \cdot 0$ & $33 \cdot 6 \pm 4 \cdot 2$ \\
\hline
\end{tabular}



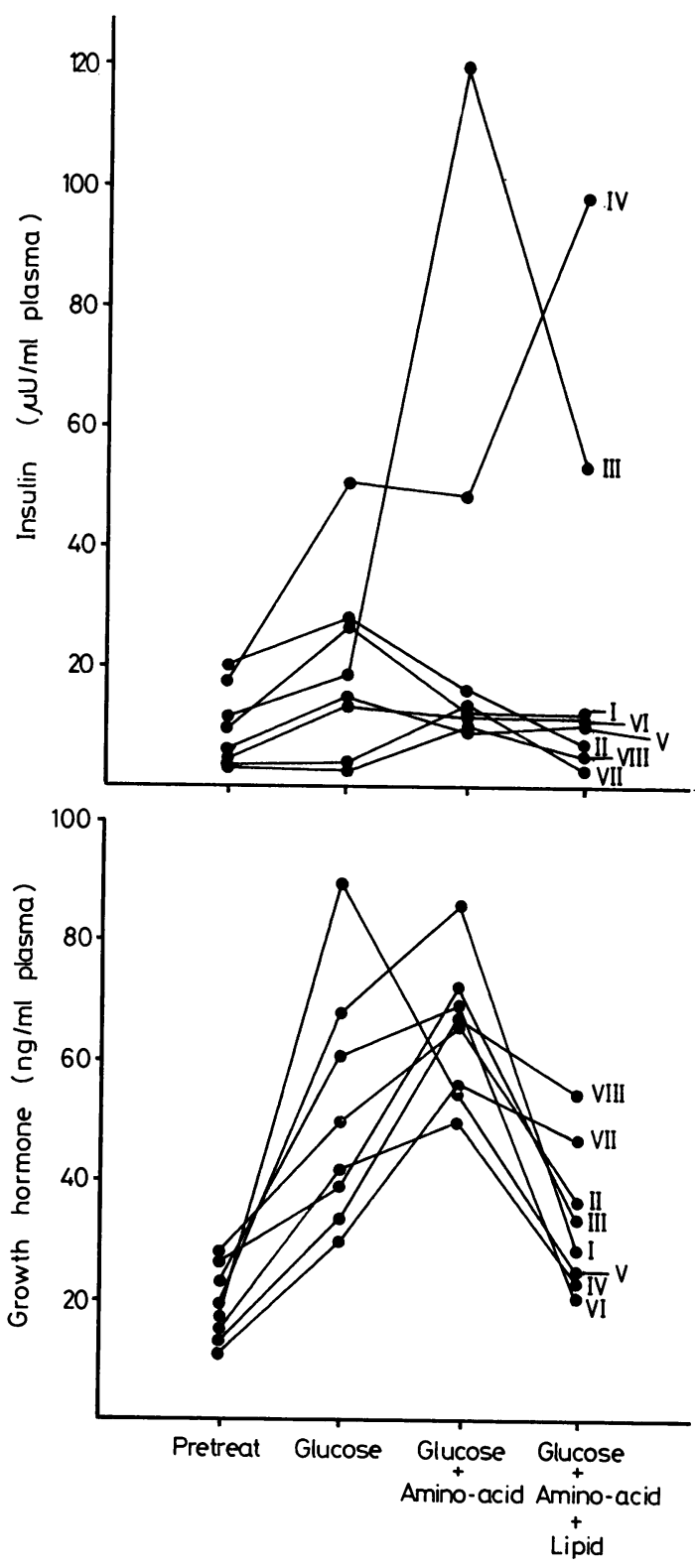

Fig. 4 Plasma insulin and growth hormone during the glucose, glucose + amino-acid, and glucose + amino-acid + lipid infusions to 8 infants.

much variation. The changes in $\mathrm{GH}$ were more uniform. The definite increase elicited by $10 \%$ glucose was followed by a further pronounced increase in response to the combined infusion of glucose and amino-acids, when addition of lipid was associated with a pronounced fall.

\section{Discussion}

It has been shown that the basal metabolic rate of small infants and the total daily heat production is considerably less (Mestyán et al., 1968; Rubecz and Mestyán, 1975) than that predicted by Gordon and Levine (1936). The three levels of caloric intake (lower, equal, and higher than the basal requirement) represented by the three infusion periods were chosen to take account of this newer information.

Changes in blood glucose and FFA. As in our previous observations (Rubecz et al., 1974) hypertonic glucose infusion caused a rise in blood glucose and a fall in plasma FFA. The fall in FFA became more pronounced by addition of the amino-acids to $10 \%$ glucose, but blood glucose was much lower. As expected, the addition of fat emulsion led to a substantial rise in plasma FFA, blood glucose remaining unchanged.

Responses in plasma amino-acids and urea level. The hypoaminoacidaemia observed by the end of the $10 \%$ glucose period is in accordance with the known interrelationship between carbohydrate and amino-acid metabolism (Munro and Thomson, 1953). The removal of amino-acids from the plasma as shown by the fall in $\alpha$-amino-nitrogen content was significant. It is noteworthy that the rise in plasma $\alpha$ amino-nitrogen after amino-acid supplementation did not exceed the fasting level. This confirms our previous finding (Rubecz et al., 1974) that administration of hypertonic glucose is an effective means of avoiding hyperaminoacidaemia, a potentially dangerous consequence of infusing amino-acid mixtures. It is also significant that the normal total plasma amino-acid level was maintained during the third 12-hour period when lipid was infused in addition to the glucose and amino-acid solution.

Supplementation with amino-acid mixture increased the plasma concentrations of most individual amino-acids. The increment was variable and seemed to reflect the composition of the amino-acid solution infused. Taurine was the only amino-acid where plasma level fell throughout the infusion periods. The infusion of lipid in addition to glucose and amino-acids altered the plasma amino-acid pattern appreciably.

The fact that blood urea was practically unchanged indicated that the amount of amino-acids infused did not cause a rate of urea production which exceeded the renal excreting capacity. In view of the possibly increased amino-acid transport to tissues due to stimulated insulin secretion (Kipnis and Noall, 1958) it appears reasonable to assume that the anabolic use of amino-acids was also improved 
when the caloric input, and hence the amino-acid load, did not exceed the maintenance energy expenditure.

Changes in plasma insulin concentration. The magnitude and time course of the individual changes in plasma insulin were found to be variable. This particularly applied to the glucose period when a marked or a moderate response was observed or sometimes none. This is consistent with reports which point out that the insulin response to a glucose load in either normally grown or growth-retarded low birthweight infants is generally poor, particularly within the first 48 hours of life (Baird and Farquhar, 1962; Milner and Hales, 1965; Grasso et al., 1968; Gentz et al., 1969). The change of plasma insulin after supplementation of glucose with amino-acids was also found to be variable: it either elicited a small rise in infants in whom glucose was ineffective, or maintained the moderate increase caused by glucose infusion. In two infants it even abolished the glucose-stimulated insulin secretion. These observations are in accordance with the observations of Pildes and Wong (1973) and with our previous study (Rubecz et al., 1974) in which administering amino-acids in addition to glucose failed to enhance insulin secretion. The low birthweight, immaturity, and pathological conditions of infants included in the previous and present studies may be a relevant factor in explaining the lack of synergism between glucose and amino-acids, and the inconsistent pattern of insulin response.

Although direct evidence for an effect of plasma FFA on insulin secretion in man is lacking, FFA have been shown to stimulate insulin secretion in the dog (Crespin et al., 1969). In the present investigations lipid did not seem to modify consistently the plasma insulin content, which either remained unchanged or fell to the fasting level. In only one infant was a further appreciable increase observed. Asch et al. (1975) reported a somewhat higher (but nonsignificant) insulin level in infants in response to a $12 \%$ glucose $+2.5 \%$ amino-acid and $10 \%$ soybean oil emulsion parenteral nutrition regimen, compared with the infusion of a mixture of $12 \%$ glucose and $2 \cdot 5 \%$ amino-acid solution.

Changes in plasma growth hormone concentration. Unlike the changes in plasma insulin, the response of GH was more uniform. In all infants but one, the response induced by $10 \%$ glucose was further increased by the supplementation with amino-acids. It is well known that in newborn infants glucose induces GH release, and amino-acids-such as arginine-are also potent stimuli (Stubbe and Wolf, 1970; Le Dune, 1972; Stubbe et al., 1973). It is interesting that further supplementation with lipid depressed GH level in most infants, in some it completely abolished the response elicited by glucose and glucose amino-acids. In adults (Blackard et al., 1971), and in rhesus monkeys (Blackard et al., 1969), the administration of corn oil emulsion and heparin suppressed insulin and arginine-induced GH secretion, and it was suggested that this effect might have been due to the FFA component. It is conceivable that in the present study the pronounced rise in plasma FFA concentration in response to lipid supplementation was mainly responsible for the inhibition of stimulated GH secretion.

Besides the changes in hormonal status the administration of lipid was also associated with alterations in the plasma aminogram: the concentrations of a number of amino-acids increased. As GH is known to augment the amino-acid uptake of the tissues (Snipes, 1968) it is reasonable to assume that the raised levels of some amino-acids in response to lipid was the result of suppression of GH secretion.

\section{Conclusions}

In view of recent estimates of the maintenance caloric need of low birthweight infants kept at a neutral temperature (Mestyán et al., 1968; Rubecz and Mestyán, 1975) the calories infused throughout the third 12-hour period were, in all probability, high enough to cover maintenance energy expenditure and low enough to avoid undesirable metabolic complications. The distribution of calories of the infusate appears also properly balanced as far as the plasma metabolic profile is concerned. The lack of pronounced biochemical distortions indicate that the pattern of substrate use is also less unphysiological than might be expected with a caloric input much exceeding heat production.

It is time to re-evaluate the energy side of parenteral feeding in low birthweight infants. An individual approach to the estimation of the caloric need for maintenance must be adopted. The components of total heat production (basal metabolic rate, specific dynamic action, activity, thermoregulatory heat production) may vary considerably according to maturity, postnatal age, feeding, and thermal conditions. These factors require individual assessment if the caloric requirement of an individual infant is to be determined.

It is also important to know whether parenteral feeding is to cover maintenance substrate and energy requirements, or is to provide additional calories for growth. In view of the usual absence of growth in low birthweight neonates during the first 10 days of life, it appears unnecessary to increase the caloric input above the maintenance requirement, otherwise most 
of the extra calories and substrates would probably be rapidly oxidised rather than incorporated in the body tissues.

\section{References}

Asch, M. J., Sperling, M., Fiser, R., Leake, R., Moore, T. C., and Oh, W. (1975). Metabolic and hormonal studies comparing three parenteral nutrition regimens in infants. Annals of Surgery, 182, 62-65.

Baird, J. D., and Farquhar, J. W. (1962). Insulin-secreting capacity in newborn infants of normal and diabetic women. Lancet, 1, 71-74.

Blackard, W. G., Boylen, C. T., Hinson, T. C., and Nelson, N. C. (1969). Effect of lipid and ketone infusions on insulininduced growth hormone elevations in rhesus monkeys. Endocrinology, 85, 1180-1185.

Blackard, W. G., Hull, E. H., and Lopez, S. A. (1971). Effect of lipids on growth hormone secretion in humans. Journal of Clinical Investigation, 50, 1439-1443.

Ceriotti, G. (1971). Ultramicrodetermination of plasma urea by reaction with diacetylmonoxime-antipyrine without deproteinization. Clinical Chemistry, 17, 400-402.

Crespin, S. R., Greenough, W. B., III, and Steinberg, D. (1969). Stimulation of insulin secretion by infusion of free fatty acids. Journal of Clinical Investigation, 48, 1934-1943.

Dalton, C., and Kowalski, C. (1967). Automated colorimetric determination of free fatty acids in biologic fluids. Clinical Chemistry, 13, 744-751.

Gentz, J. C. H., Warrner, R., Persson, B. E. H., Cornblath, M. (1969). Intravenous glucose tolerance, plasma insulin, free fatty acids, and beta-hydroxybutyrate in underweight newborn infants. Acta paediatrica Scandinavica, 58, 481490.

Gordon, H. H., and Levine, S. Z. (1936). Respiratory metabolism in infancy and in childhood. XVIII. The respiratory exchange in the premature infant's basal metabolism. American Journal of Diseases of Children, 52, 810-830.

Grasso, S., Messina, A., Saporito, N., and Reitano, G. (1968). Serum-insulin response to glucose and aminoacids in the premature infant. Lancet, 2, 755-757.

Hales, C. N., and Randle, F. J. (1963). Immunoassay of insulin with insulin-antibody precipitate. Biochemistry, 88, 137-146.

Huckabee, W. E. (1958). Relationships of pyruvate and lactate during anaerobic metabolism. I. Effects of infusion of pyruvate or glucose and of hyperventilation. Journal of Clinical Investigation, 37, 244-254.

Hunter, W. M., and Greenwood, F. C. (1962). A radioimmunoelectrophoretic assay for human growth hormone (abstract). Biochemical Journal, 85, 39P-40P.

Kipnis, D. M., and Noall, M. W. (1958). Stimulation of amino acid transport by insulin in the isolated rat diaphragm. Biochimica et biophysica acta, 28, 226-227.

Laurell, S., and Tibbling, G. (1967). Colorimetric microdetermination of free fatty acids in plasma. Clinica chimica acta, 16, 57-62.

Le Dune, M. A. (1972). Intravenous glucose tolerance and plasma insulin studies in small-for-dates infants. Archives of Disease in Childhood, 47, 111-114.

Mestyán, J., Járai, I., and Fekete, M. (1968). The total energy expenditure and its components in premature infants maintained under different nursing and environmental conditions. Pediatric Research, 2, 161-171.

Milner, R. D. G., and Hales, C. N. (1965). Effect of intravenous glucose on concentration of insulin in maternal and umbilical cord plasma. British Medical Journal, 1, 284-286.

Munro, H. N., and Thomson, S. W. T. (1953). Influence of glucose on amino acid metabolism. Metabolism, 2, 354-361.

Pildes, R. S., and Wong, P. W. K. (1973). Plasma insulin in intravenous glucose and amino-acid infusion. New England Journal of Medicine, 288, 914-915.

Pryce, J. D. (1967). A simple rapid method for determining glucose in blood or plasma. Analyst, 92, 198.

Rubecz, I., Mestyán, J., Soltész, Gy., and Horváth, M. (1974). Metabolic and hormonal effects of alternate infusion of hypertonic glucose and Aminosol-glucose in premature infants. Acta paediatrica Academiae Scientiarum Hungaricae, 15, 301-321.

Rubecz, I., and Mestyán, J. (1975). The partition of maintenance energy expenditure and the pattern of substrate utilization in intrauterine malnourished newborn infants before and during recovery. Acta paediatrica Academiae Scientiarum Hungaricae, 16, 335-350.

Rubinstein, H. M., and Pryce, J. D. (1959). The colorimetric estimation of alpha-amino nitrogen in tissue fluids. Journal of Clinical Pathology, 12, 80-84.

Snipes, C. A. (1968). Effects of growth hormone and insulin on amino acid and protein metabolism. Quarterly Review of Biology, 43, 127-147.

Stubbe, P., and Wolf, H. (1970). Glucosebelastungen und Arginin-Infusionen bei Neugeborenen. Einfluss auf Wachstumshormon, Blutzucker, Fettsäuren, und Glycerin. Klinische Wochenschrift, 48, 918-923.

Stubbe, P., Leititis, J., Leppla, W., and Wolf, H. (1973). Provocation of growth hormone and insulin secretion in full term infants small for gestational age (abstract). Pediatric Research, 7, 58.

Correspondence to Professor J. Mestyán, University Medical School of Pécs, Department of Paediatrics, 7623 Pécs, József Attila U7, Hungary.

Received 22 August 1978 